Musicalité de la parole, mise en musique du texte. Fonctions de la $<$ poésie musicale > et de sa mise en musique dans la tragédie en musique

\author{
Gallusser, Lion
}

DOI: https://doi.org/10.15203/ATeM_2020

Posted at the Zurich Open Repository and Archive, University of Zurich ZORA URL: https://doi.org/10.5167/uzh-197898

Journal Article

Published Version

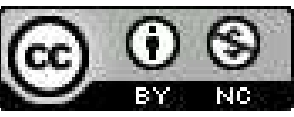

The following work is licensed under a Creative Commons: Attribution-NonCommercial 4.0 International (CC BY-NC 4.0) License.

Originally published at:

Gallusser, Lion (2020). Musicalité de la parole, mise en musique du texte. Fonctions de la $<$ poésie musicale > et de sa mise en musique dans la tragédie en musique. ATeM Archives of Text and Music Studies, $(5,2): 1-23$.

DOI: https://doi.org/10.15203/ATeM_2020 


\title{
Musicalité de la parole, mise en musique du texte. \\ Fonctions de la ‘ poésie musicale > et de sa mise en musique dans la tragédie en musique
}

\author{
Lion GALLUSSER (Zurich) ${ }^{1}$
}

\section{Summary}

Lully's and Quinault's tragédie en musique raises the question of the linkages and coordination between two creative processes in this unique genre. On the one hand, music derives its legitimacy to a large extent from poetry. On the other, a metrical and musical analysis expanding on the account of Le Cerf de la Viéville demonstrates a range of particularities in the structuring of poetry in these works, which at times adapts to pre-existing music, and more frequently anticipates it. Mise en musique, the setting of words to music, is generally in line with poetic dynamics. However, the use of its own means allows it to introduce additional semantic content, marking the strengthening of its autonomy within the artistic system.

La tragédie en musique - créée par Jean-Baptiste Lully (1632-1687) et le librettiste Philippe Quinault (1635-1688), à l'apogée de la politisation de l'art voulue par Louis XIV (1638$1715)^{2}$ - suppose un texte, puis sa mise en musique. Au-delà du procédé créateur, l'articulation entre ces deux composantes devait renforcer la légitimité d'un genre destiné à être perçu comme une tragédie mise en musique, et, par conséquent, comme un genre sérieux (cf., aussi pour la perception des tragédies en musique en tant qu' œuvres littéraires, Girdlestone 1972, 3-29). Chacun des deux créateurs se devait de faire un pas envers l'autre. S'il était attendu du compositeur qu'il sache parfaitement mettre en musique le texte écrit par le librettiste, ce dernier, de son côté, devait être capable d'écrire un texte qui se prête à cette mise en musique. Le cas de Lully et Quinault montre que ces deux artistes se complétaient justement dans ces tâches, ce qui fut essentiel à leur réussite artistique.

Nombreux sont les enjeux en ce contexte. Quelles étaient les caractéristiques d'une bonne poésie lyrique, telle qu'elle était attendue dans le livret ? Et comment ce texte était-il mis en musique par le compositeur ? Cet article a pour but de répondre à ces questions en montrant quelques caractéristiques de la poésie musicale ${ }^{3}$ et de sa mise en musique, à l'aide de plusieurs exemples tirés de différentes tragédies en musique de Lully et Quinault. Ses 
résultats permettront de mieux connaître les exigences poétiques de Quinault et aideront à comprendre comment Lully, lorsqu'il se faisait compositeur d'une tragédie en musique, mettait en musique les paroles.

Il est d'abord nécessaire de donner un bref aperçu de l'histoire et de la conception de la tragédie en musique afin de comprendre la légitimation toute littéraire de ce genre. Le livret sera ensuite discuté, en tant qu'élément central de toute la tragédie en musique. La partie essentielle de cet article traitera finalement des composantes de la poésie musicale et de sa mise en musique, ainsi que de l'influence de cette poésie sur les structures musicales.

\section{La tragédie en musique comme genre littéraire}

Lorsque Cadmus et Hermione est présenté pour la première fois à l'Académie royale de $\mathrm{Mu}$ sique en 1673, Lully et Quinault, ses créateurs, peuvent se féliciter d'avoir créé la ‘ tragédie en musique ', qui restera le genre prééminent de l'opéra français jusqu’à la chute de l'Ancien Régime. Encouragés par le Roi Soleil, qui s'était prononcé pour la création d'un opéra explicitement français après avoir assisté à plusieurs opéras italiens aux alentours de 1650 (cf. Leopold 2004, 174-179)4, Lully et Quinault créent une nouvelle forme d'art qui rassemble plusieurs genres déjà existants, et typiquement français - tels que le Ballet de Cour, ou l'Air de Cour dans une ouvre d'art qui doit être grandiose et totale, représenter la gloire et le pouvoir de la France de la monarchie absolue. ${ }^{5}$ Ce genre nouveau visait à éblouir le spectateur par de multiples canaux : son ouïe par la musique, son esprit par la poésie, ses yeux par la mise en scène et les machines (on n'hésitait pas, par exemple, à faire descendre sur la scène des divinités ; cf. Leopold 2004, 174-179).

La tragédie en musique se voulait donc au premier rang de la politisation des arts prévue par Louis XIV. Ainsi, elle allait plus loin que les autres genres lyriques français, grâce à sa mise en rapport avec la forme d'art alors la plus prestigieuse, la tragédie classique, alors en plein essor - c'est l'époque de Pierre Corneille (1606-1684), de Thomas Corneille (1625-1709) et de Racine (1639-1699). En se voulant concurrente artistique directe de la tragédie déclamée, la tragédie en musique s'appropriait, adaptait et modifiait les propriétés fondamentales de celleci. Un genre lyrique nouveau trouvait donc sa légitimité dans le recours au théâtre classique (cf. Gier 1998, 57s). La tragédie en musique reprend le moule de la tragédie déclamée, ce qui se remarque notamment dans la structuration en cinq actes et dans le respect des éléments déclamatoires par la mise en musique (nous reviendrons sur ce point plus en détail). Mais elle ne respecte cependant pas toutes les règles du théâtre classique - unité de lieu, de temps et d'action, pour une intrigue vraisemblable dont le déroulement logique est déterminé par la nécessité ; elle inverse ces composantes pour mieux les assimiler à un cadre qui lui soit propre, ${ }^{6}$ et la condition essentielle au fonctionnement de cette < inversion > est le merveilleux (pour le merveilleux, cf. art. "Merveilleux », Sadie 1992, 345). Celui-ci permet de soustraire l'intrigue de la tragédie en musique à la conception qui préside à celle de la tragédie déclamée et permet de légitimer l'univers au sein duquel se déroule l'action des opéras. Le merveilleux 
autorise la multiplication des personnages surnaturels, tels que les dieux, et des ‘ divertissements ', qui constituent des parties autonomes, au sein desquelles le ralentissement de l'action ouvre au déploiement de la danse et de la musique, typiquement lors de scènes de fête ou de foule. La notion justifie également le fait que les personnages soient continuellement en train de chanter - ce qui ne serait évidemment pas acceptable dans la logique du théâtre classique ; elle autorise, encore, la violation des règles de l'unité classique, l'intrigue de la tragédie en musique n'étant pas tenue de se dérouler au même endroit, ni en vingt-quatre heures, ni de se concentrer sur une seule action. Dans le cadre du merveilleux, il est tout à fait légitime que les lieux changent rapidement (les divertissements se déroulant par exemple souvent dans des endroits exotiques), que l'action expose analeptiquement les antécédents, et que s'étalent des intrigues secondaires. Toutefois, malgré les écarts par rapport à la norme classique que permet le recours au merveilleux, la dramaturgie de la tragédie en musique suit, comme la tragédie classique, les règles de la nécessité et de la vraisemblance. L'action doit, dans le monde merveilleux de la tragédie en musique, rester logique, claire, cohérente et vraisemblable par rapport à son propre cadre de référence (cf. Girdlestone 1972, 8).

Cette conception de la tragédie en musique comme un ‘ négatif `, ou l'inversion (cf. note 5) chantée de la tragédie déclamée les met directement en relation. Les divergences pouvant toutes être ramenées à l'introduction du merveilleux, la tragédie en musique se voit comme un genre lyrique aux fondations littéraires. Un exemple frappant de la manière dont la tragédie en musique trouve sa légitimité et tente d'accéder au même statut que la tragédie classique par le recours à une origine littéraire, tout en assumant son autonomie, se retrouve au début de la toute première tragédie en musique, Cadmus et Hermione (1673), déjà évoquée. ${ }^{7}$ La présence d'abord uniforme de l'alexandrin régulier dans les premiers vers donne - malgré l'absence de rimes suivies - l'impression d'un renvoi à la tragédie parlée :

PREMIER PRINCE TIRIEN (Recueil général des opéras 1703, vol. 1, 159)8

Quoi, Cadmus, fils d'un roi qui tient sous sa puissance 12

Les bords féconds du Nil et les Climats brûlez; $\quad 12$

Cadmus, après deux ans loin de Tir écoûlez, $\quad 12$

Étranger chez les Grecs, n'a point d'impatience 12

De revoir un Pays dont il est l'espérance? 12

Et laisse sans regrets tant de cœurs désolez? $\quad 12$

\section{LES DEUX PRINCES TIRIENS}

Nous suivons vos destins par tout sans résistance ; 12

Faudra-t-il que toûjours nous soyons exilez? 12

\section{CADMUS}

J'aimerois à revoir les lieux de ma naissance ;

Mais avant que je puisse en goûter la douceur,

12

J'ai juré d'achever une juste vengeance. 
Mais, dès la première réplique du premier prince, le mètre varie rapidement, alternant entre l'alexandrin, l'octosyllabe et l'hexasyllabe (Recueil général des opéras 1703, vol. 1, 160) :

\section{PREMIER PRINCE TIRIEN}

Et cependant, Seigneur,

Vous laissez en ces lieux languir vostre grand cœur.

\section{CADMUS}

Après avoir erré sur la Terre et sur l'Onde

Sans trouver Europe ma Sœur ;

Après avoir en vain cherché son ravisseur,

Le ciel termine ici ma course vagabonde ;

Et c'est pour obéir aux oracles des Dieux

Qu'il faut m'arrêter en ces lieux.

Cette alternance des mètres est continuée dans la suite de la tragédie. Bien que se retrouvent encore des alexandrins, la constante variété des mètres éloigne décidément le texte de la tragédie déclamée, étant donné que le chant demande au mètre d'être plus flexible dans la tragédie en musique. Louverture initiale en alexandrins renvoie donc à la tragédie classique, et permet de s'inscrire dans la continuité de ses vers nobles, bien que la variation par rapport à ceux-ci soit, dès les premières rimes, perceptible dans le schéma des rimes. Une fois la référence et la légitimation qui en découle établies, l'irrégularité du mètre, confirmant celle de la rime, montre nettement la nouveauté de la tragédie en musique, qui, plutôt qu’une trop simple ‘ mise en musique ' propose en fait une alternative à la tragédie classique.

Cette conception toute littéraire de ce nouveau genre se retrouve dans la grande importance que Lully et Quinault accordaient à la mise en musique du texte. Le poids accordé à celle-ci se manifeste dans le raffinement et le grand travail effectué par les deux artistes à cet égard, toujours dans une optique de légitimation. Le recours à une déclamation mi-chantée, telle que l'on supposait qu'elle se pratiquât dans l'antiquité, permettait de présenter - notamment par le récitatif français - une nouvelle approche imitative du théâtre antique (cf. Couvreur 1992, 306). En résumé, il était donc, d'une part, possible de justifier les divergences par rapport au théâtre classique grâce à l'introduction du merveilleux. D'autre part, au-delà de la légitimité que permettait la filiation avec la tragédie déclamée, on visait à entrer en concurrence avec elle en lui proposant une alternative. À l'instar des Italiens qui fondaient leur opéra sur le recitativo, qui, pour sa part, intégrait les efforts de la Camerata florentine sous Giovanni de' Bardi (1534-1612), pour le fameux recitar cantando qu'auraient exercé les anciens dans leurs théâtres, la tragédie en musique prétendait proposer un renouvellement meilleur de la pratique des anciens que celui de la tragédie classique (cf. Couvreur 1992, 306). Jamais le théâtre déclamé n’aurait-t-il pu aussi bien correspondre au théâtre des anciens, ni dans sa déclamation, ni dans sa conception trop restreinte, excluant toute autre forme d'art. C'est notamment par l'introduction du chœur en tant qu'élément musical, pratique bannie du théâtre classique, que 
la nouvelle tragédie en musique se présentait comme la tragédie véritable (sur le théâtre français et le chœur, cf. Louvat-Molozay 2008). En introduisant la musique comme véhicule de tout procédé dramatique sur scène, la tragédie en musique se fondait sur un art qui avait déjà beaucoup évolué aux alentours de 1600, par le passage de la polyphonie à la monodie, c'est-àdire de la musique à plusieurs voix égales à une musique avec une ligne mélodique sur un fond harmonique dominé par la basse. De 1650 à 1670, la période immédiatement précédente à la première tragédie en musique, cette " révolution musicale " (Duron 2004, 140) s'était imposée dans la plupart des compositions nouvelles. Celles-ci présentaient donc de nouvelles caractéristiques musicales, dont la plus remarquable est peut-être l'organisation du temps par la tonalité. Ce procédé aboutit à des constructions musicales rationalisées, à une nouvelle forme musicale dont - pour le moins en France - le souci de grandes structures logiques et claires est analogue à la conception rationalisée des jardins d'André Le Nôtre (1613-1700) ou de la façade symétrique du Louvre de Claude Perrault (1613-1688). Le développement musical s'inscrit ainsi dans la pensée socioculturelle de la France du XVII ${ }^{\mathrm{e}}$ siècle.

\section{La fonction du livret}

Le livret est une composante essentielle de la tragédie en musique. Non seulement il comporte le contenu dramatique - l'action en tant que telle -, mais encore il sert de base conceptuelle à l'opéra. En tant que structure permettant la coordination entre tous les éléments de la tragédie en musique, le livret esquisse celle de l'œuvre finale en intégrant toutes ses composantes (cf. Gumbrecht 1986, 18). La coordination et la conception commune de ces composantes étaient d'autant plus importantes que - ainsi qu'il a été évoqué au début de cet article - la tragédie en musique a expressément été conçue comme une œuvre d'art totale qui incorporait tous les arts dramatiques pour pouvoir représenter et faire rayonner la gloire de la France. Malgré la complexité de leur interaction, nous proposons de regrouper ces composantes en quatre catégories, selon leurs fonctions. D'abord, les composantes purement textuelles sont considérées comme porteuses de l'action. Il s'agit là du texte, dans son sens propre, qui se prête en premier lieu à l'analyse littéraire, ou à l'analyse socioculturelle de l'opéra, dans le cadre d'une interprétation littéraire et historique, en tant qu'il représente les idéologies et les esthétiques de la France absolutiste. Ensuite, les composantes musico-textuelles - telles que les récitatifs, les airs et les chœurs - résultent soit de la mise en musique du texte du livret, soit, inversement et dans une deuxième sous-catégorie, de paroles mises sur de la musique préexistante. Comme il sera montré dans la suite, les paroles de certains des passages d'une tragédie en musique notamment dans les divertissements - n'ont été écrites qu’après la composition de la musique des airs, danses et chœurs qui leur correspondent. Troisièmement, les ouvertures, les préludes et ritournelles, les entr'actes et les danses sans paroles représentent les composantes purement musicales. Toute cette musique est sans texte. Finalement, il y a les composantes visuelles, dont sont les danses - c'est-à-dire les mouvements des danseurs -, les chorégraphies, les gestes des personnages, et les décors - y compris les costumes et les machines. 
Notre aperçu montre que la poésie destinée à être mise en musique n'est qu'un élément parmi ceux qui constituent notre genre. Toutefois, la mise en musique y occupe une place prépondérante parce qu'elle représente - comme il a déjà été expliqué - l'élément fondamental de sa légitimation. En conséquence, il était d'une toute première importance de lui porter une attention diligente, notamment lorsqu'elle est celle du " corps de l'opéra " (Le Cerf de La Viéville 1705, 218) - pour reprendre les termes de Jean-Laurent Le Cerf de la Viéville (16741707), grand thuriféraire de Lully - qui comprend presque toute l'action de la tragédie en musique, sauf le divertissement. C'est dans ce " corps de l'opéra " qu'est employé le nouveau récitatif, dont il était, comme nous l'avons vu, attendu qu'il revienne à la déclamation des anciens, mais qui, dans les faits, s'orientait plutôt à une déclamation à voix rehaussée, telle qu'elle était pratiquée dans les théâtres de l'époque.'

Il est donc évident qu'une bonne collaboration entre le poète et le compositeur était indispensable pour établir une mise en musique adaptée à toutes les exigences du genre nouveau. Nous savons que Lully et Quinault collaborèrent de manière très étroite, pour chacune de leurs treize tragédies en musique qui se sont succédé de manière presque annuelle, jusqu'en $1686 .{ }^{10}$

Faisons brièvement le point sur cette collaboration (cf. Gier 1998, 64) qui arriva à une méthode de travail plus au moins standardisée. Une fois le sujet de l'opéra arrêté par le roi lui-même, Quinault en faisait d'abord une esquisse de la conception et rimait les vers de tous les passages de l'action. Ensuite, Lully examinait les vers de ce qui constituait donc le « corps d'opéra "; s'il en désapprouvait certains, il les renvoyait à Quinault, qui en proposait de nouveaux. Une fois Lully d'accord avec l'ensemble, celui-ci - selon la Viéville - « lisait [une scène] jusqu'à la savoir presque par cœur [puis] il s'établissoit à son clavessin, chantoit et rechantoit les paroles, battoit son Clavessin et faisoit une basse continuë. Quand il avoit achevé son chant, il se l'imprimoit tellement dans la tête, qu'il ne s'y seroit pas mépris d'une Note " (Le Cerf de La Viéville 1705, 215). Lully cherchait donc une correspondance musicale ‘ naturelle $\iota^{11}$ à la poésie de Quinault par le biais du chant, qui respectait les exigences de la langue. Pour les divertissements en revanche, donc pour toutes les parties de l'opéra où l'action se ralentit ou s'interrompt en faveur du merveilleux (des fêtes populaires, des danses, etc.), Quinault proposait une esquisse rudimentaire de l'action à Lully sans rimer aucun vers. En se fondant sur cette proposition, Lully composait la musique - il s'agit avant tout de danses, de chœurs et d'airs, jamais de récitatifs. Le Cerf de la Viéville résume ainsi le procédé :

$[\mathrm{P}]$ our les divertissemens, Lulli faisoit les airs d'abord, à sa commodité \& en son particulier. Il y falloit des paroles. Afin qu'elles fussent justes, Lulli faisoit un canevas de vers, \& il en faisoit aussi pour quelques airs de mouvement. Il apliquoit lui-même à ces airs de mouvement $\&$ à ces divertissemens, des vers, dont le mérite principal étoit de quadrer en perfection à la Musique, \& il envoyoit cette brochure à Quinaut [sic], qui ajustoit les siens dessus. (Le Cerf de La Viéville 1705, 218s)

Il revenait donc en ce cas à Quinault de trouver des vers à mettre sur les notes, et les rythmes, de Lully. 


\section{Fonctions de la poésie musicale et de sa mise en musique}

\section{La poésie au carcan des divertissements}

La division entre le " corps de l'opéra " et les " divertissements » se retrouve dans la disposition métrique des livrets des différentes tragédies en musique. Les passages d'un divertissement avaient à ‘ mettre en texte ` des notes préexistantes dont les rythmes sont normalement réguliers et se répètent souvent selon les exigences de la danse ou du chœur que Lully avait choisi. Il s'ensuit que les paroles qui leur sont agrégées sont également régulières et leur correspondent en longueurs, accents, et en rimes. Ce procédé peut être illustré par un chœur issu de la troisième tragédie en musique de Lully et Quinault. Dans la scène septième du troisième acte de l'opéra Thésée de 1675, «Les Habitans des Enfers expriment [dans un chœur] la douceur qu'ils trouvent dans les ordres que Médée leur donne de donner des frayeurs et de faire de la peine à Églé " (Lully 2010, 24). Il s'agit d'un chœur typique, dont la structure est la suivante : premier air (à danser), chœur I, second air, chœur II. ${ }^{12}$ Tandis que les deux airs sont différents l'un de l'autre, la musique du chœur est répétée à l'identique. Pourtant cette dernière se révèle comme une variation du second air dont la mesure à trois temps détermine les chœurs, ce qui augmente la cohésion musicale de la scène. Bien que les textes initiaux varient entre les chœurs, ils sont construits - du point de la vue de la métrique de la même façon. La structure des six premiers vers (qui sont à chaque fois répétés dans leur chœur) est la suivante : $:^{13}$

On nous tourmente

Sans cesse aux Enfers,

Que l'on ressente

Nos feux et nos fers.

Tout doit se troubler,

Tout doit trembler.

[...]

On nous déchaîne,

Suivons nos fureurs ;

Dans notre peine

Troublons tous les Cours.

Un grand désespoir,

Est doux à voir.

\section{rime nombre de syllabes}

a $4(+1)$

B 5

a $4(+1)$

B 5

C 5

C 4

d $4(+1)$

E 5

d $4(+1)$

E 5

F $\quad 5$

F 4

\section{Accents fixes (en gras et soulignés)}

Accents secondaires (soulignés; proposition en rapport avec la mise en musique) 
La partition nous montre que, pour les premiers quatre vers, la mesure à trois temps est parfaitement respectée par la longueur des vers et avant tout par leurs accents qui tombent tous sur un temps musical fort. Qui plus est, les deux quatrains respectent également la carrure musicale de deux fois quatre mesures : premièrement par leur unité sémantique et syntaxique de deux vers, deuxièmement par leurs rimes croisées qui renforcent le sentiment de parallélisme. Les vers 5 et 6 des deux chœurs suivent également la disposition musicale que Lully choisit pour le chour : mais, tandis que les vers 5 sont encore des pentasyllabes respectant par leurs accents les temps forts de la mesure 3/4, l'ambiguïté métrique des vers 6 permet de tenir les mesures correspondantes. Ces dernières (mes. 120-122 et mes. 178-180) ne s'accordent que superficiellement à un rythme en 3/4, car elles forment une hémiole, dont Lully se sert souvent à la fin d'une carrure musicale pour produire un effet de ralentissement : grâce à un changement de mesure, passant de $3 / 4$ à 3/2, ces deux mesures - commençant sur la troisième noire de la mesure 120 et allant jusqu'au début de la mesure 122 - sont musicalement considérées comme une (et non plus comme deux). Dans cette nouvelle mesure binaire, un temps fort tombe sur chaque blanche, et le vers 6 propose des accents qui peuvent convenir (sans musique il est moins facile de décider sur quelles syllabes ces accents tombent). On remarque avant tout que, dans le premier chœur, la première syllabe "Tout ", dans le vers 5 , n'est pas accentuée, alors qu' elle l'est dans le vers suivant. L'indétermination de l'accentuation du vers français permet à Quinault de recourber les vers en question afin de faire correspondre leurs accents à ceux de la musique. Ce n'est donc qu'à travers la musique qu'on peut savoir que les avant-dernières syllabes des vers 6 ne sont pas accentuées (" trem » et "à » tombent sur une croche qui est inaccentuée parce qu'elle sert d'anticipation pour la note finale accentuée). Les vers 5 et 6 par ailleurs, sont, à la simple lecture sans musique, métriquement très inhabituels pour l'époque - ou bien un ralentissement comme dans l'hémiole, dans le passage brusque d'un vers de cinq à un vers de quatre syllabes masculines ; les vers précédents n’étaient pas habituels, eux non plus, à la poésie écrite, respectant l'alternance des genres (féminin/masculin), mais en réduisant le nombre de syllabes des vers féminins. ${ }^{14}$ Il s'ensuit que la poésie a ici pour fonction de suivre la musique, par un texte et une métrique qui s'adapte aux caractéristiques musicales, ce qui aboutit à une poésie qui prétend être régulière, mais dont le but principal est de respecter les temps musicaux - et qui va jusqu'à ignorer les limites habituelles de la métrique (l'exemple musical qui suit est tiré de Lully 1688, consultable en ligne) : 
LES O MBRES.

25

On nous tourmente Sansceffe aux Enfers, Que l'on reffente nos felix \& nos fers. Tour

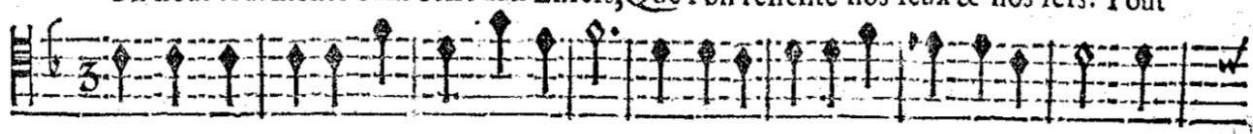

On nous tourmente Sansceffe aux Enfers, Quel'on reffente nos feux \& nos fers. Tour

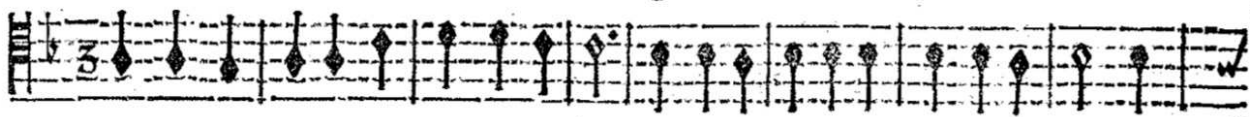

On nous tourmente Sans ceffe aux Enfers, Que l'on reffente nos fenx \& nos fers. Tour

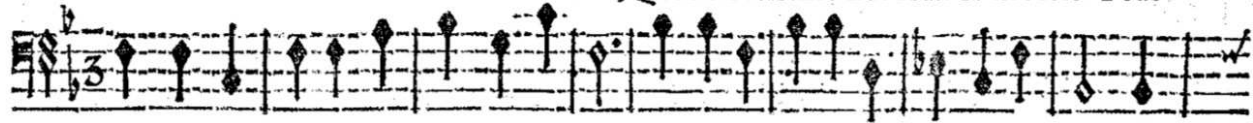

On nous tourmente Sans ceffe aux Enfers, Que l'on reffente nos feux \& nos fers. Tout

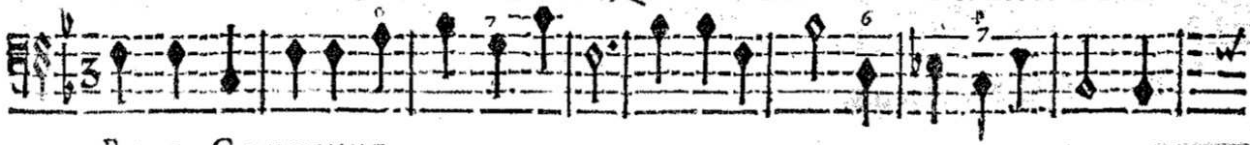

BASSECONTINUE.

246 doil fe trotbler, Tout doir trembler. LaColere Ne laiffe jamais Nos Coxurs en

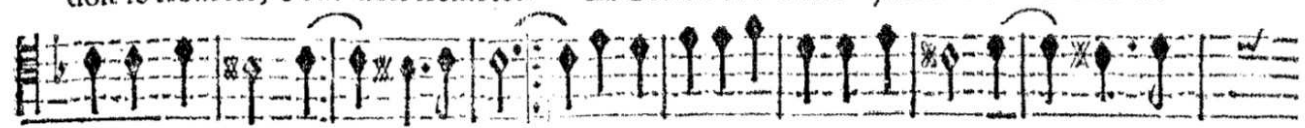
doit fe troubler, Tout doit trembler. La Colere Ne laille jamais Nos Caurs en

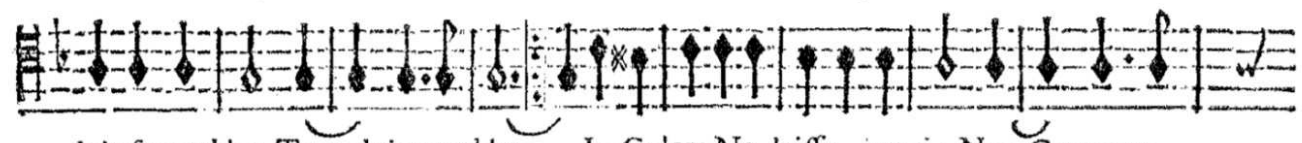
doit fe troubler, Tout doit tremblet. La Colere Ne laiffe jamais Nos Coeurs en

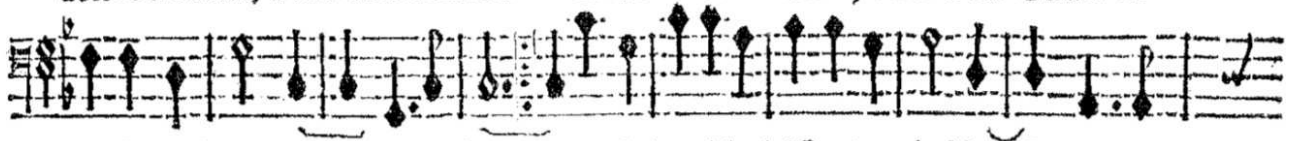
doit fetroubler, Tout doittrembler. $\quad \mathrm{L}_{\mathrm{a}}$ Colere Ne laife jamais Nos Cours en

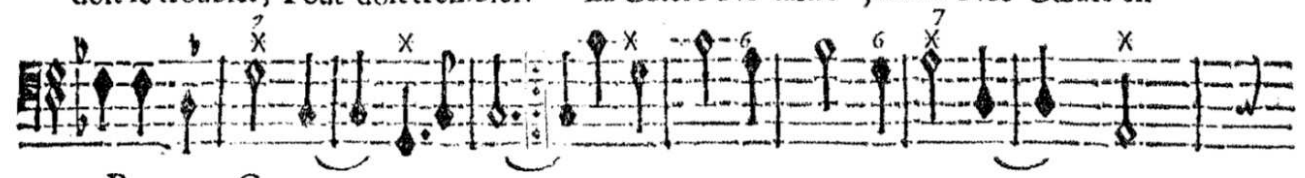
BASSE-CONTINUE. 


\section{LES OMBRES.}

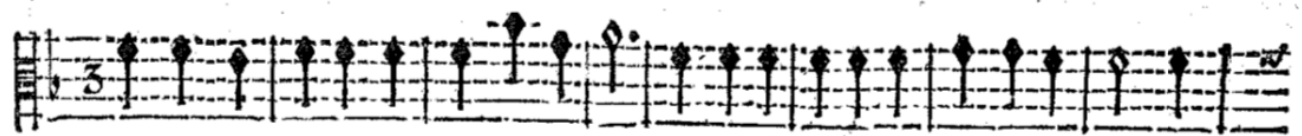

On nous déchaîne, Suivons nos fureurs, Dans noftre peine Troublós tous les cœurs, $\mathrm{U}_{\mathbf{n}}$

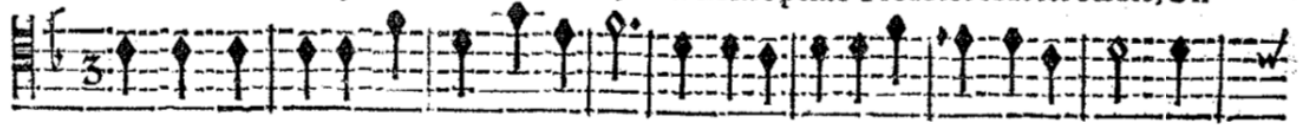

On nous déchaîne, Suivons nos fureurs, Dans noftre peine Troublös zous les cœurs, $U_{n}$

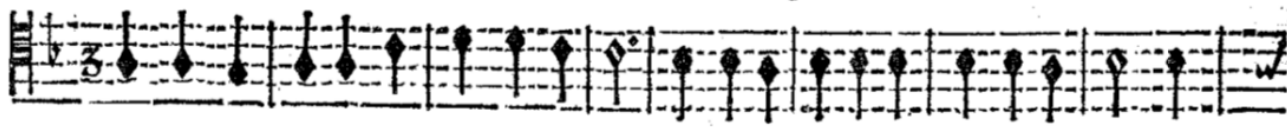

On nous déchaîne, Suivons nos fureurs, Dans noftre peine Troublǒs tous les cœeurs, $\mathrm{Un}_{\mathrm{n}}$

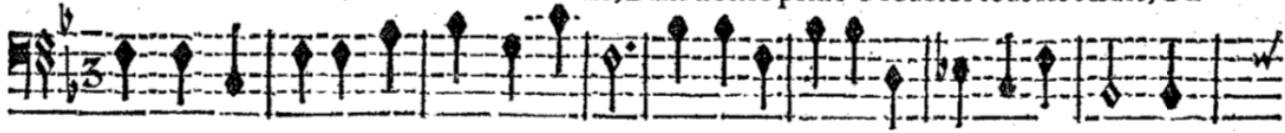

On nous déchaîne, Suivons nos fureurs. Dans noftre peine Troublös tous les cceurs, Un

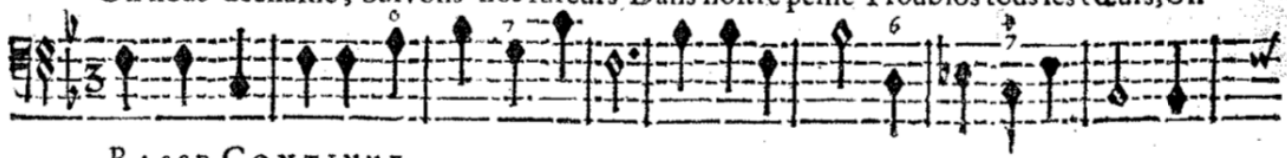

BASSECCONTINUE.

$24^{8}$ THESEE TRAGEDIE.

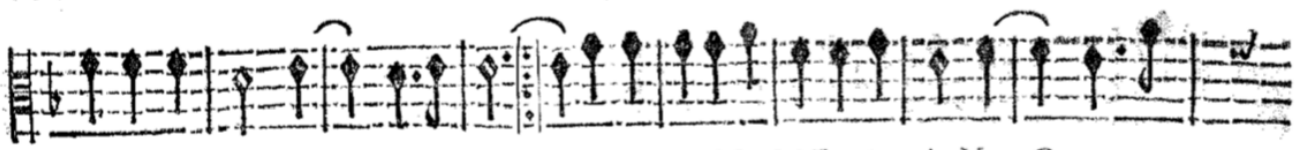

grand defefpoir Eft doux à voir. LaColere Ne laifle jamais Nos Cocurs en

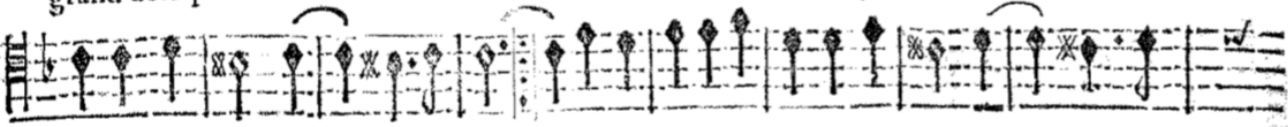

grand defefpoit Eft doux à voir. LaColere Ne laifle jamais Nos Cocurs en

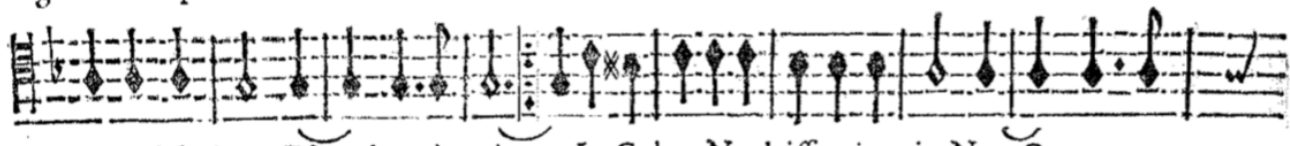

grand defefpoir Eft douxà voir. La Colere Ne laiffe jamais Nos Cocurs en

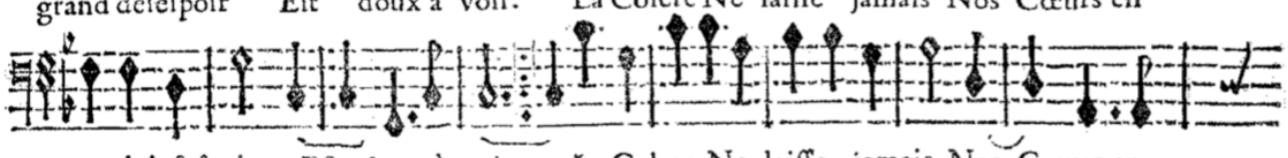

grand defefpoir Eft doux à voir. La Colere Ne laiffe jamais Nos Cours en

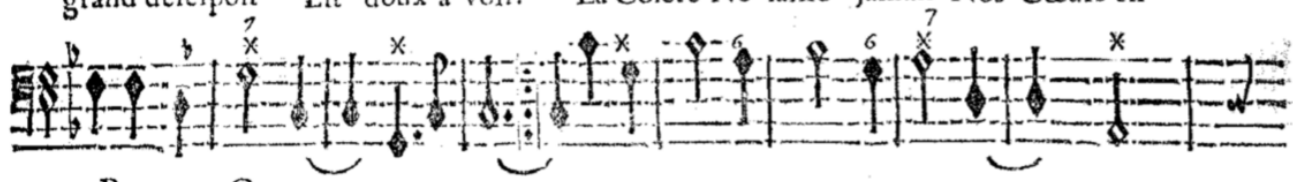
BASSE-CONTINUE.

Figure 1. Jean-Baptiste Lully, Thésée, Paris : Christophe Ballard, 1688 


\section{L'absence de régularité métrique dans le corps de l'opéra}

La poésie des passages du corps d'opéra, dans lequel se développe l'action, est, à la différence des divertissements, marquée par l'absence de régularité métrique. Pour décrire cela, référons-nous à la scène deuxième du premier acte de Atys, la quatrième tragédie en musique de Lully et Quinault, créée en 1676. Dans cette scène, Idas s'adresse à son ami Atys en voulant lui faire découvrir son amour pour la nymphe Sangaride (cité d’après le Recueil général des opéras 1703, 381) :

$\begin{array}{llll}\text { Atys, ne feignez plus, je sçay vostre secret. } & \text { a } & 12 & 6(2+4)+6 \\ \text { Ne craignez rien, je suis discret, } & \text { a } & 8 & 4+4 \\ \text { Dans un bois solitaire \& sombre, } & \text { b } & 8 & 3+5(3+2) \\ \text { L'indifferent Atys se croyoit seul un jour ; } & \text { c } & 12 & 6(4+2)+6(3+3) \\ \text { sous un feüillage espais où je resvois à l'ombre, } & \text { b } & 12 & 6(4+2)+6(4+2) \\ \text { Je l'entendis parler d'amour. } & \text { c } & 8 & 4+4\end{array}$

Quinault a écrit des vers libres classiques, c'est-à-dire des vers mêlés - à ne pas confondre avec les ‘ vers libres > modernes, connus depuis le XIX' siècle (cf. par exemple Bray 1951, 456) - pour ce passage (cf. Berger 1998, 112).${ }^{15}$ Ce type de vers se distingue en effet par l'absence d'une régularité métrique, et il présente des rimes différentes sans schéma fixe, des accents variables et des longueurs différentes. À cause de sa flexibilité décontractée, ce type de vers semble davantage refléter une déclamation libre et se prête parfaitement à une mise en musique variée. Remarquons que la mélodie et le rythme de la musique coïncident avec celui d'une déclamation naturelle. Le principe fondamental qui est à la base de ce procédé est qu'une structure accentuelle cohérente et mise en valeur par la métrique poétique doit être mise en relief par le positionnement sur les débuts de mesure ou sur les temps musicaux forts. Les notes musicales - les valeurs rythmiques et les mélodies attribuées aux différentes paroles - dépendent de la prononciation et de la sémantique des mots.

Sachant que la fonction de la mise en musique est de traduire la poésie en une déclamation musicalement intensifiée, on remarque que le récitatif respecte un rythme qui peut se retrouver dans les paroles. La musique peut traduire le rythme des paroles en des notes de différentes longueurs dont la valeur rythmique résulte des longueurs des notes voisines ${ }^{16}$ :

L'indifférent Atys se croyait seul un jour ;

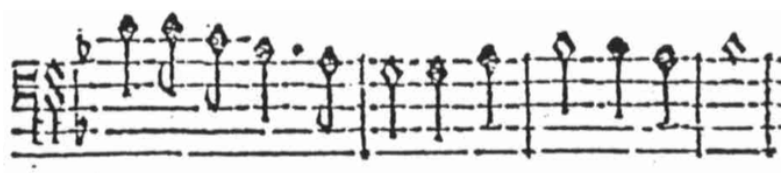

Lindifferent Atys fe croyoit feul un jour;

Figure 2. Jean-Baptiste Lully, Atys. Tragédie mise en musique, Paris : Ballard, 1679 
Quinault composait habituellement des < vers libres > d'une grande variabilité rythmique, ainsi que des sous-sections rythmiques de courte longueur (cf. Norman, 325s), ce qui permettait à Lully de les mettre aisément en musique d'une façon variée. La répartition des vers en plusieurs unités rythmiques de longueur différente pourrait donc témoigner d'une sensibilité de Quinault au rythme musical donné aux paroles par Lully, dont il anticipe la mise en musique. Or, cette mise en musique peut également rendre plus intense l'émotion qu'expriment les énoncés ; à cet égard, les croches et doubles-croches de la première intervention d'Idas semblent refléter son agitation : «Atys, ne feignez plus, je sçay vostre secret. »

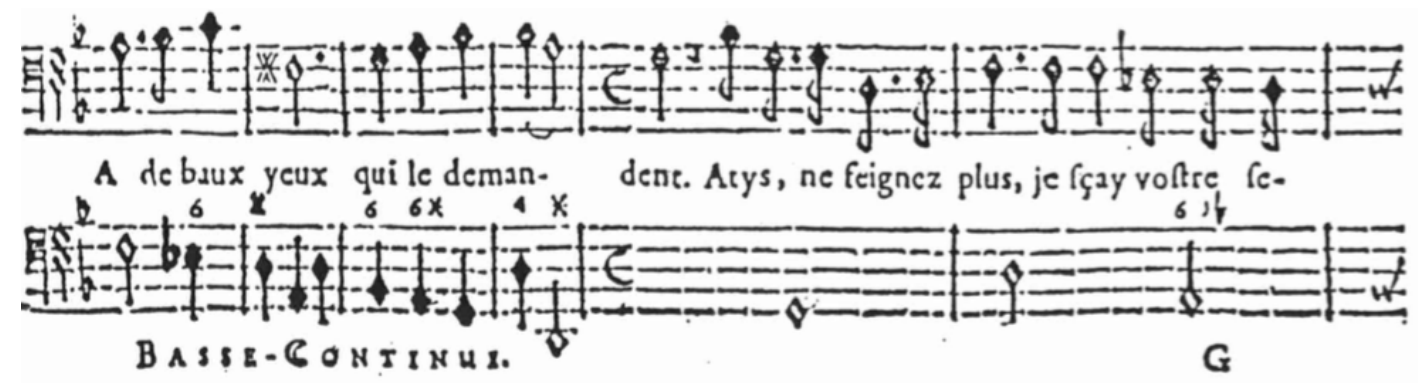

Figure 3. Jean-Baptiste Lully, Atys. Tragédie mise en musique, Paris : Ballard, 1679

Par la suite, c'est encore une fois l'agitation qui semble exprimée par le débit accéléré d'Idas, mais pour aboutir cependant à une autre émotion. Par crainte, probablement, de se montrer déloyal envers son ami - d'où, encore une fois, l'agitation des notes rapides -, Idas veut tranquilliser Atys : « Ne craignez rien, je suis discret. » Cette insistance sur sa loyauté se manifeste musicalement dans le ralentissement subit et la cadence sur le mot « discret » - toute rapidité et toute agitation semblent ultimement se dissoudre.

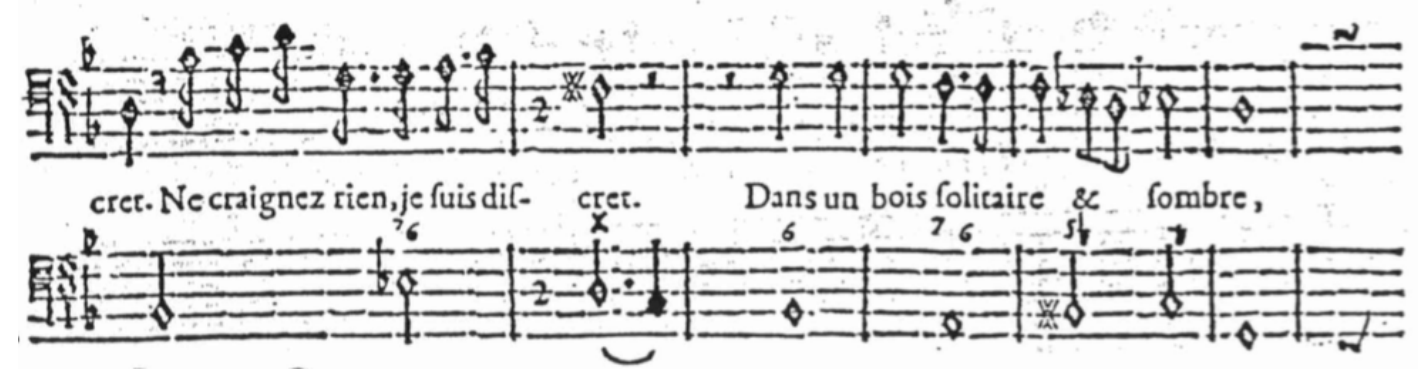

Figure 4. Jean-Baptiste Lully, Atys. Tragédie mise en musique, Paris : Ballard, 1679

Au vers suivant, Idas dit avoir vu Atys en proie à l'amour dans un «bois solitaire et sombre ". Une lecture à voix haute du vers semble déjà inciter à un débit moins rapide que dans les vers précédents. Quinault lie des voyelles graves et de longues quantités syllabiques ("Dans ", " bois ", " solitaire ", "sombre ") à la divergence sémantique entre l'agitation d'Idas et son 
récit. Or, ledit récit est admirablement mis en paroles, ces syllabes ainsi que l'allitération en ( $s$ ) ("solitaire et sombre ») contribuant à l'impression d'un bois calme et obscure.

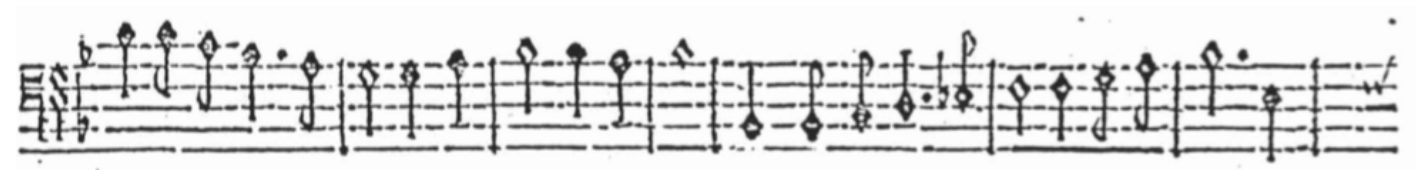

L'indifferent Atys fe croyoic feul un jour; Sous un feüillage efpais où je refrois ̀̀
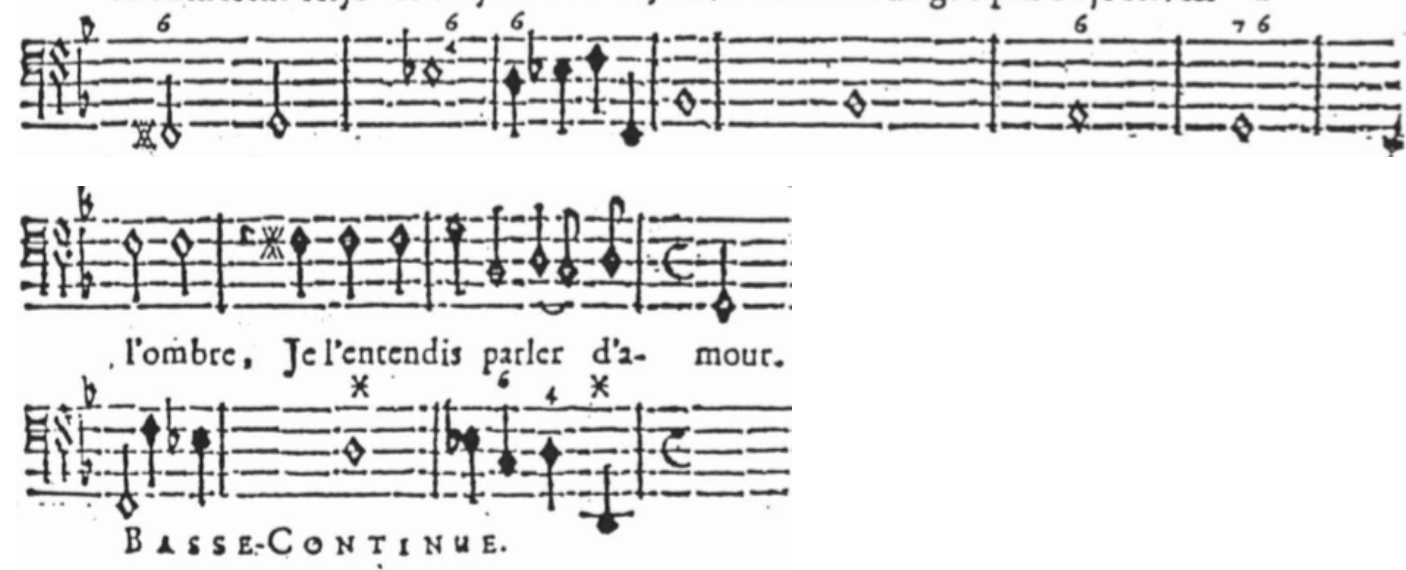

Figure 5. Jean-Baptiste Lully, Atys. Tragédie mise en musique, Paris : Ballard, 1679

Lully était donc susceptible de se fonder sur des paroles très ‘ musicales `, capables d'illustrer le signifié par leur sonorité. Sa mise en musique traduit cette musicalité poétique en musique en renforçant toutes les voyelles longues par des notes relativement longues ("Dans », " bois ", " solitaire ", " sombre ») ${ }^{17}$, qui se distinguent des notes voisines par une durée plus longue. De surcroît, et parallèlement au débit de la poésie, la vitesse musicale a également baissé : si l'agitation d'Idas est d'abord marquée par vingt notes pour quatre mesures («Atys, ne feignez plus, je sçay vostre secret. / Ne craignez rien, je suis discret »), dix notes, pour quatre mesures, renvoient au calme de la forêt ("Dans un bois solitaire \& sombre»).

\section{Linfluence de la poésie sur les structures musicales}

Suite à l'examen de ces deux exemples, nous pouvons constater que la poésie conçue pour être mise en musique met à la disposition du compositeur un texte d'une grande variété rythmique et métrique, sur laquelle il peut s'appuyer pour écrire la musique correspondante, déjà anticipée par le librettiste. Mais la poésie musicale peut aussi donner lieu à des structures plus complexes encore que le simple récitatif. En effet, la tragédie en musique est caractérisée par le fait que le texte est mis en musique dans une multitude de formes, dont les différences qui les séparent et les changements intervenus entre elles ne sont pas toujours 
faciles à définir. Contrairement à l'opéra italien de la deuxième moitié du XVII ${ }^{\mathrm{e}}$ siècle, qui standardisait de manière conséquente la différence entre aria - pour illustrer les émotions et les moments de réflexion - et recitativo - servant à faire avancer l'intrigue -, la tragédie en musique déploie plusieurs sortes de récitatifs et d'airs qui se chevauchent et s'entremêlent. Bien qu'il ne soit pas possible de prédire la mise en musique à partir de la poésie musicale, plusieurs structures purement musicales sont influencées par elle. C'est notamment le cas du grand monologue de la sorcière Armide, au début du troisième acte de l'opéra du même nom (1686), lui-même basé sur l'épopée de La Jérusalem délivrée du Tasse (1544-1595). Armide se plaint de l'amour qu'elle éprouve pour Renaud, le meilleur des chevaliers chrétiens $:^{18}$

A Ah! si la liberté me doit être ravie,

Estce à toi d'être mon vainqueur?

Trop funeste ennemi du bonheur de ma vie,

Faut-il que malgré moi tu règnes dans mon cœur ?

B

Le désir de ta mort fut ma plus chère envie,

Comment astu changé ma colère en langueur ?

En vain de mille amants je me voyais suivie,

Aucun n'a fléchi ma rigueur.

Se peut-il que Renaud tienne Armide asservie !

A Ah! si la liberté me doit être ravie,

Estce à toi d'être mon vainqueur ?

Trop funeste ennemi du bonheur de ma vie

Faut-il que malgré moi tu règnes dans mon cœur ? a

b

12

8

a

b

d

c $\quad 12$

d 8

c $\quad 12$

12

12

8

12

12
$126(1+5)+6(3+3)$

$83+5$

$126(3+3)+6(3+3)$

$126(2+4)+6(2+4)$

$126(3+3)+6(4+2)$

$126(2+4)+6(3+3)$

$26(2+4)+6(4+2)$

$2+3+3$

$6(3+3)+6(3+3)$

$6(1+5)+6(3+3)$

$3+5$

$6(3+3)+6(3+3)$

$6(2+4)+6(2+4)$

\section{Accents fixes (en gras et soulignés)}

Accents secondaires (soulignés; proposition en rapport avec la mise en musique)

La structure métrique de ce poème peut être résumée en un ABA. La mise en musique respecte cette forme (A : mes. 8-15 ; B : mes. 15-24 ; A : mes. 25-32), mais y ajoute un prélude de huit mesures qui viennent présenter la mélodie et le rythme du chant de la partie A. Tandis que les dessus de violon anticipent le chant d'Armide à l'identique, les autres instruments ne renforcent que quelques tournures rythmiques et mélodiques. Le prélude purement musical (mes. 1-8) est ainsi influencé par le rythme des paroles des premiers quatre vers (les exemples musicaux sont tirés de Lully 1686, consultable en ligne) : 


\section{ACTE TROISIESME}

\section{SCENE PREMIERE}

Le Theatre change \& represente un Desert. ARMIDE, seule.

3-1 ARMIDE : Ah ! si la liberté me doit estre ravie
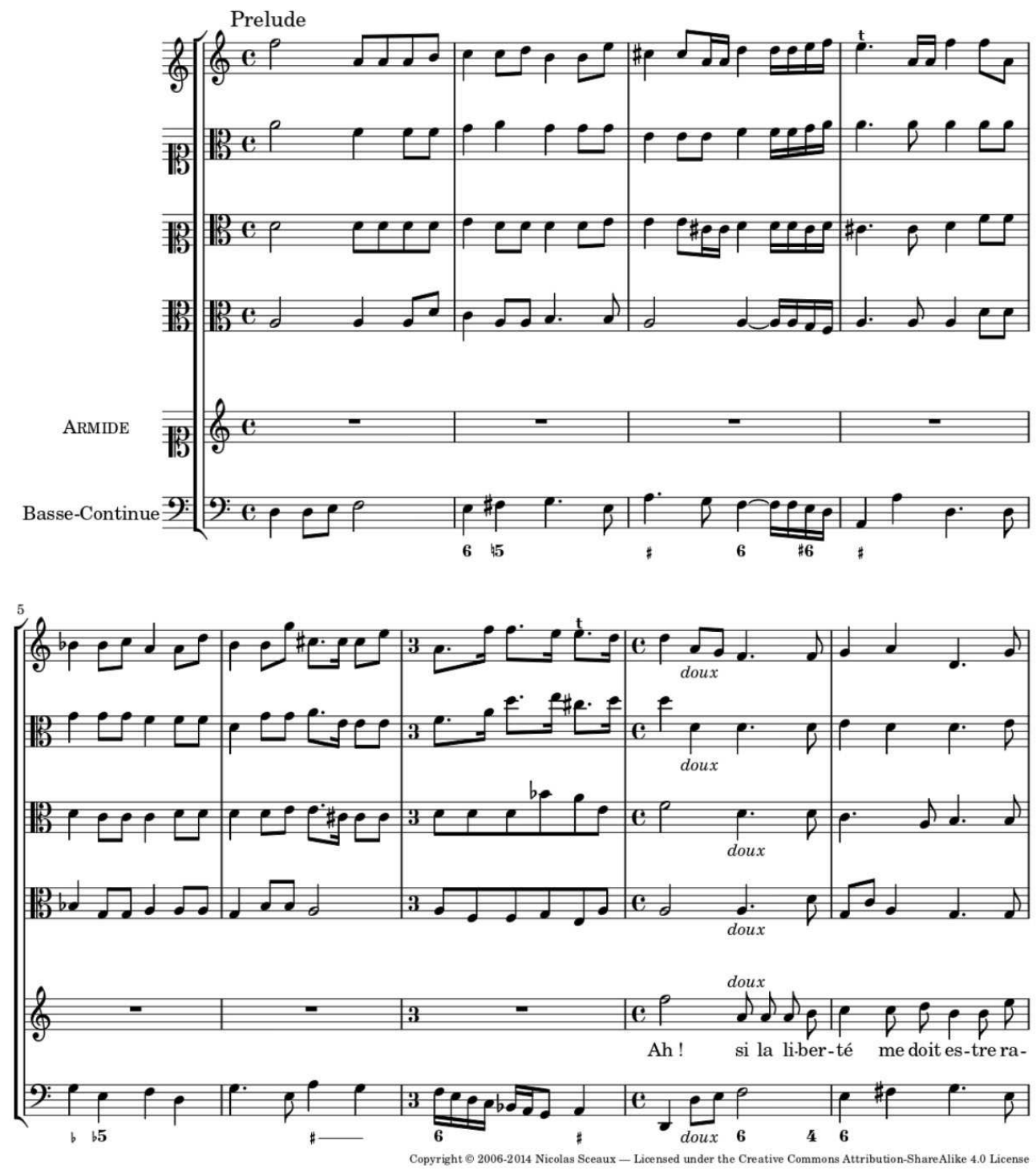

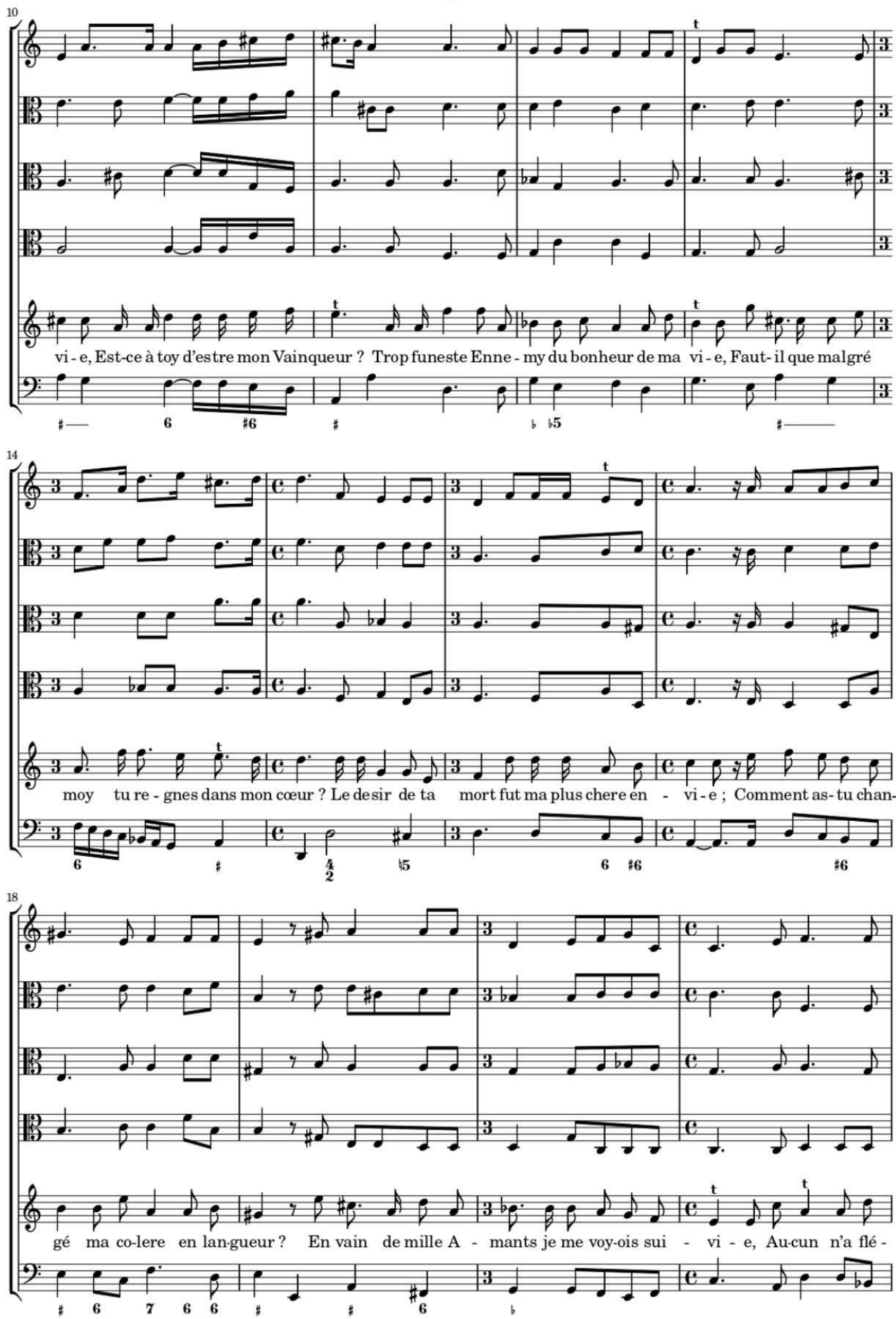

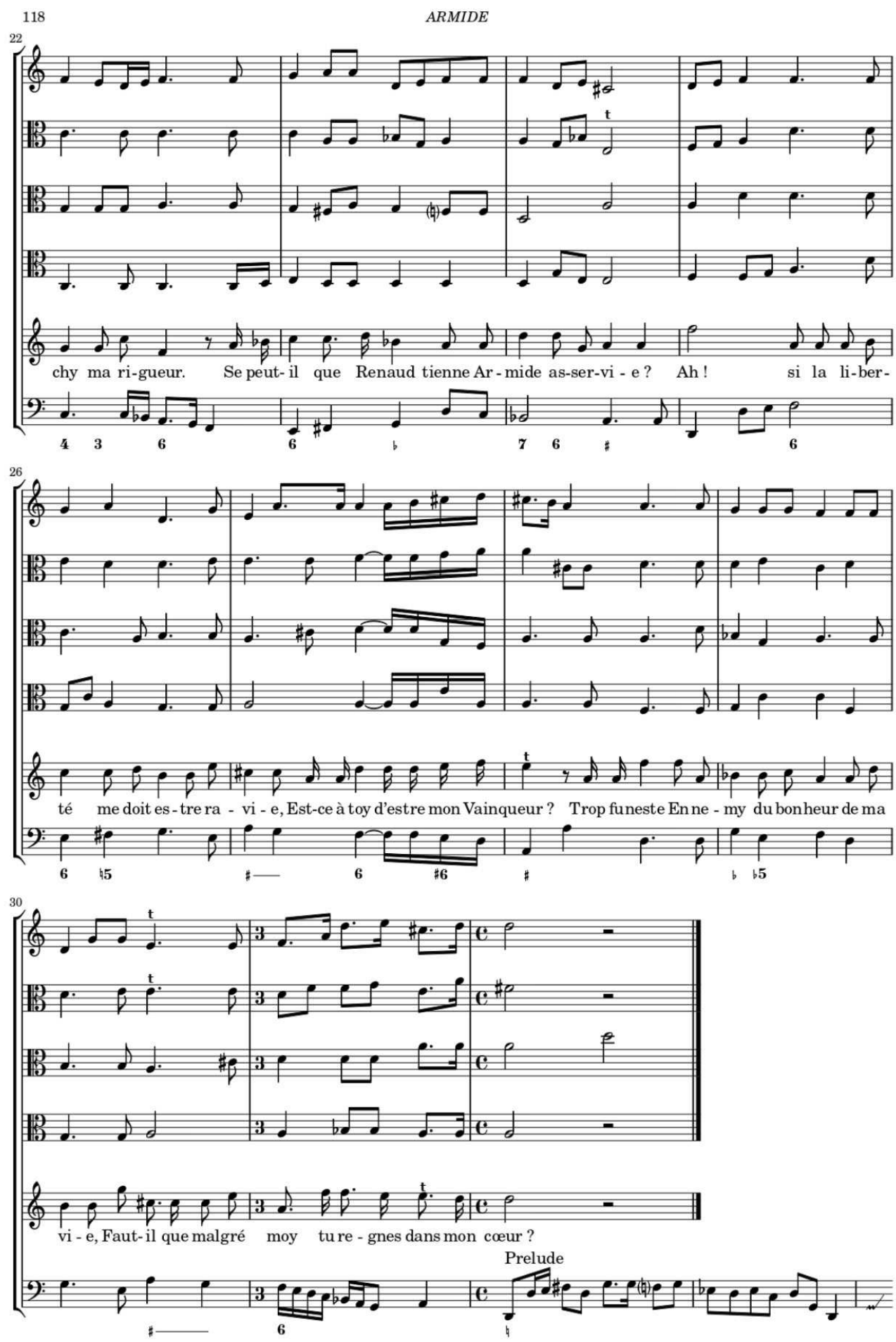

Figure 6. Jean-Baptiste Lully - Philippe Quinault, Armide, Paris : Ballard, 1686 
Quinault a, encore une fois, composé des vers libres au sein desquels plusieurs sous-sections rythmiques peuvent être définies, puis aisément mariées avec la musique : le premier alexandrin peut être réparti en quatre sous-sections; la première, la plus courte, n'est qu'une exclamation ("Ah!») et se prête parfaitement à la première note longue pathétique qui la distingue, isolée sur un $f a$ qui est la sixte du la suivant (mes. 8 de l'exemple précédent). Dans le deuxième vers, l'octosyllabe introduit d'autres rythmes encore, en deux élans (que la sémantique et la syntaxe combinées semblent déjà identifier), dont le second introduit une figure de doubles-croches, caractéristique (mes. 10s). Le troisième vers met une fois encore en valeur une certaine disposition rythmique, et reprend, en l'inversant, la sixte du premier vers (mes. 11) ; or, la reprise de cette sixte qui vient d'être mise en rapport avec la douleur de l'exclamation ("Ah!»), permet, dans la mise en musique, d'adjoindre à cette souffrance la mention d'un "Trop funeste ennemi ", combinant les deux passages. Ainsi, poursuivant la réflexion, nous concluons que le prélude uniquement musical ne fait pas qu'anticiper la voix d'Armide en tant que masse sonore, influencée par certains rythmes de la déclamation. Non, la musique du prélude véhicule déjà l'intuition de la souffrance d'Armide, ce dont l'auditeur se rend définitivement compte lorsqu'il écoute les paroles de son chant.

Mais la musique ne sert pas uniquement à renforcer la sémantique des vers par une connotation des motifs musicaux. Son organisation peut également renforcer la structure poétique. Si l'on a déjà remarqué que le dialogue reprend musicalement la structure tripartite de la poésie, la disposition harmonique des différentes parties montre que les ‘ tons > - harmonie, dans le langage de l'époque - employés correspondent aux différents niveaux sémantiques. Alors que les parties A - au sein desquelles Armide se plaint de son sort par autant de questions que de signes de désespoir - sont harmoniquement fermées en un ré mineur, la partie B part de ce ré mineur, et change plusieurs fois de ‘ ton > pour, finalement, arriver sur le la majeur (mes. 24), lui-même la dominante du ré mineur de la partie $\mathrm{A}$ ou, respectivement, de sa reprise. La partie B, dans laquelle Armide fait un bref résumé des événements qui ont permis à Renaud de la séduire, est donc harmoniquement dépendante de la partie A. Bien que cette dépendance entre les deux parties poétiques se retrouve déjà dans le texte, elle reste implicite et ne se révèle qu'à travers l'interprétation. La mise en musique, en revanche, souligne clairement que la souffrance de la partie A est le résultat du passé qui est le sujet de la partie $B$, grâce à un enchaînement harmonique des deux parties. Ceci est d'autant plus fascinant que la relation dominante-tonique n'était alors qu'en train de se consolider, de s'instaurer en tant que tension, dominant le développement du temps musical, dans le contexte de la "révolution musicale " (Duron 2004, 140) de la deuxième moitié du XVIIe siècle dont il a déjà été question. Le contraste sémantique entre les deux parties est cependant renforcé par la mise en musique de la partie $B$ en un récitatif flexible - on y constate beaucoup de mesures différentes, des changements fréquents de tonalités et un chant qui est plus proche de la déclamation - ce qui permet de penser qu'Armide cherche à expliquer son destin. La mise en musique de la partie A est, a contrario, plutôt statique - sans changements de mesures, avec un accompagnement instrumental important et dans une tonalité qui reste assez stable -, ce 
qui contribue à donner l'impression d'une Armide qui s'abandonne au désespoir, se noyant dans le chant, dans l'air pathétique qu'elle chante avec tout l'orchestre.

\section{Conclusion}

Nous avons observé combien la ‘ poésie musicale ` (cf. note 2) et sa mise en musique ont été essentielles à la légitimation de la tragédie en musique. Cependant, au-delà des témoignages de Le Cerf de la Viéville sur la collaboration de Lully et Quinault, l'approche analytique précise les caractéristiques qui sont propres à la poésie musicale et à sa mise en musique. La première est disposée métriquement afin de faciliter la seconde. Les ‘ vers libres > de l'époque et leur flexibilité, leur possible partition en des sous-sections rythmiques reconnaissables, et une richesse sonore qui peut permettre la connotation par les phonèmes - comme, par exemple, par les syllabes longues - y contribuent également. Si, dans le corps de l'opéra ce sont les vers libres qui dominent, dans les divertissements, les vers s'adaptent surtout à la musique préexistante : à la limite de la régularité métrique, ils se conforment aux propriétés musicales. Dans ce dernier cas, on pourrait donc parler d'une ‘ mise en parole `, ou d'une ‘ mise en poésie > de la musique.

Dans le cadre de la légitimation de la tragédie en musique, de son ambition d'être un genre au prestige égal à celui de la tragédie déclamée, la fonction de la mise en musique est de renforcer ou d'accentuer - par ses moyens propres - certaines caractéristiques de la déclamation tout en respectant la métrique et la sémantique des paroles. Mais la musique peut, d'ellemême, également intégrer davantage de contenu sémantique. Ainsi, si les motifs et la tonalité peuvent apporter davantage de sens au texte, la conséquence en est que la musique en tant que telle devient - en gagnant plus d'autonomie dans le système artistique - porteuse de sens.

L'approche analytique s'est révélée être une méthode utile à la connaissance de certaines exigences poétiques et musicales nécessaires à une mise en musique. Tandis qu'un bon poète lyrique devait à la fois être capable d'écrire des vers adaptés aux caractéristiques de la poésie musicale et de comprendre les procédés essentiels de la mise en musique de ses paroles, le compositeur devait, de son côté, avoir une connaissance approfondie de la poésie lyrique et de ses propriétés potentiellement musicales. Il est indiscutable que Lully et Quinault connaissaient ces enjeux, et les attentes de leur collaborateur respectif. C'est peut-être là que réside la clé du succès de leur travail. ${ }^{19}$

\section{Notes}

1 Lion Gallusser est collaborateur scientifique au département de musicologie de l'Université de Zurich. Après des études en musicologie, littérature et linguistique françaises à l'Université de Zurich, à l'Université François Rabelais de Tours et au Centre d'études supérieures de la Renaissance (CESR), il vient d'achever sa thèse sur la conception des tragédies en musique entre 
Lully et Rameau (1687-1733). Il s'intéresse au statut et à l'esthétique de l'opéra français dans le contexte de la politique culturelle des XVII ${ }^{e}$ et XVIII ${ }^{e}$ siècles, notamment à travers le rapport entre musique et paroles.

2 L'histoire de la création de l'opéra français a fait l'objet de plusieurs études. On ne renvoie ici qu'à l'article fondamental de Jean Duron (1991), dans lequel sont présentés la conception et les paramètres de la tragédie en musique.

3 On entend par ‘ poésie musicale > la poésie écrite pour être mise en musique.

4 Leopold énumère les différents opéras italiens représentés à Paris.

5 Une bonne vue d'ensemble de la tragédie en musique se trouve dans l'indispensable ouvrage de James R. Anthony sur la musique baroque française (Anthony 1997 ; cf. également Schneider 2016).

6 Pour les réflexions sur la tragédie en musique en tant qu’inversion de la tragédie déclamée, cf. Kintzler (1991).

7 L'auteur doit l'incitation à cette réflexion aux remarques précieuses de Prof. Dr. Anselm Gerhard.

8 Cité d'après le Recueil général des opéras, vol. 1, 159s; les chiffres indiquent les nombres d'accents par vers ; un auditeur habitué de la tragédie classique se serait sans doute attendu à des distiques (rimes suivies) et aurait certainement remarqué, par rapport à la tragédie parlée, la différence dans la disposition des rimes de ce début d'une tragédie en musique.

9 Pour le récitatif, Lully s'inspirait en effet de la déclamation nuancée de la fameuse actrice La Champmeslé (Marie Chevillet, 1642-1698) qu’il avait souvent vue sur la scène de la Comédie de l'hôtel de Bourgogne (cf. Anthony 1997, 106).

10 Entre 1673, l'année de la création de Cadmus et Hermione, et 1686, l'année de la création d'Armide, le dernier opéra de Lully et Quinault, une pause n'intervient qu'entre 1678 et 1681 - suite à une disgrâce temporaire de Quinault après la représentation de Isis (1677), après laquelle il lui fut, entre autres, interdit d'écrire des livrets (cf. Schneider 2016a ; sur la disgrâce de Quinault après Isis cf. Norman 2009, 179-182).

11 Le terme « naturelle > est ici compris comme désignant une ‘ bonne > correspondance entre texte et musique. Il faut ajouter que le « naturel > devient, dans le discours esthétique à partir de 1700 environ, la catégorie fondamentale pour l'évaluation d'une composition musicale. Lecerf de la Viéville, par exemple, défend la mise en musique ‘ naturelle > de Lully, qui suivrait bien la déclamation et le texte, contre les ‘ exagérations > de la musique italienne, dont François Raguenet a souligné les qualités, et dont les ‘ roulades > contrediraient le bon sens. Raguenet (avec son Parallèle des Italiens et des François en ce qui regarde la musique et les opéra de 1702 et la Défense de cet ouvrage de 1705) et Lecerf de la Viéville (avec sa Comparaison de la musique italienne et de la musique françoise[,] où, en examinant en détail les avantages des Spectacles, \& le mérite des Compositeurs des deux nations[,] On montre quelles sont les vrayes beautés de la Musique de 1704 et 1705) se livraient une véritable querelle sur les valeurs de la musique française et italienne. Ce qui est « naturel `, il faut le souligner, dépend fortement de jugements sociopolitiques; le « naturel ? n'est pas une catégorie fixe.

12 La scène entière se trouve dans Lully (2010, 252-259). 
13 Dans l'aperçu suivant, les accents tombent tous sur les vers soulignés. Les vers qui ne sont que soulignés représentent les accents secondaires, les vers qui sont soulignés et en gras représentent les accents fixes (cf. Buschmeier 1995, 173).

14 Ce qui revient à nier la règle fondamentale de la poésie métrique française, selon laquelle les vers français sont métriquement équivalents s'ils ont le même nombre de syllabes, comptées jusqu’à la dernière voyelle masculine (toute voyelle sauf le schwa), et non pas jusqu'à la dernière voyelle, quelle qu'elle soit. Même pour des vers mêlés, ces vers sont à la limite des habitudes métriques.

15 Berger parle de "vers libres ", mais entend bien évidemment des vers libres classiques et non les ( vers libres ) de la fin de siècle.

16 Cet extrait musical ainsi que les suivants sont tous tirés de la partition imprimée par Christophe Ballard en 1679 (Lully 1679, 49s).

17 Cf. Bacilly (1679). Dans cette source indispensable pour la réalisation du chant à cette époque qu'écrit Bertrand de Bacilly (1621-1690), théoricien et maître de musique au temps de Lully et Quinault, on trouve plusieurs indices qui indiquent que les syllabes mentionnées sont considérées comme longues. Sans l'expliquer plus avant, nous nous permettons de renvoyer aux pages correspondantes chez Bacilly pour « Dans » (336s ou 357), « bois » (356) et « sombre » (348). La prononciation du mot ‘ solitaire > est, elle, plus délicate : alors que la syllabe ‘ tai > est considérée comme longue (388s), les deux premières voyelles devraient être courtes. Cependant, par l'allongement de « so > par une noire pointée et l'attribution d'une croche plus courte à ‘ li , Lully accentue métriquement la sonorité du < o > qui est d'une certaine façon reprise par la voyelle nasale dans " sombre ».

18 Cité d'après l'édition critique du livret (Lully 2003, 16).

19 De grandes parties de cet article, qui représente une version écrite et élaborée de mon exposé tenu lors du colloque, correspondent à l'article Eberhard 2016, qui résumait ma présentation en se basant sur mon manuscrit pour la communication lors du colloque.

\section{Bibliographie}

Anthony, James R. : French Baroque Music From Beaujoyeulx to Rameau. Revised and Expanded Version. Portland (Oregon): Amadeus Press, 1997.

Bacilly, Bertrand de : L'art de bien chanter. Paris : [ chez l'autheur»], 1679.

Berger, Christine : "Armide zwischen Resignation und Rache - Quinault und Lully zwischen Abschied und Ambition ». In : Archiv für Musikwissenschaft 55 (1998), 110-131.

Bray, René : "L'Introduction des vers mêlés sur la scène classique ». In : Publications of the Modern Language Association 66,4 (1951), 456-484.

Buschmeier, Gabriele : "Glucks ‘ Armide >-Monolog, Lully und die ‘ Philosophes > . In : Beer, Axel / Lütteken, Laurenz (éds) : Festschrift Klaus Hortschansky zum 60. Geburtstag. Tutzing : Hans Schneider, 1995, 167-180.

Couvreur, Manuel : Jean-Baptiste Lully. Musique et dramaturgie au service du prince. Bruxelles : Marc Vokar, 1992. 
Duron, Jean : "L'^ instinct > de M. de Lully ». In : Christout, Marie-Françoise et al. (éds) : La tragédie lyrique (Les carnets du théâtre des Champs-Elysées). Paris : Cicéro, 1991, 65-119.

Duron, Jean : «Pierre Perrin, un ‘ Virgile François > ? . In : Conihout, Isabelle de / Gabriel, Frédéric (éds) : Autour de ' La Chartreuse > de Pierre Perrin, poème imprimé par Pierre Moreau en 1647. Paris/Chambéry : Bibliothèque Mazarine/Édition Comp'Act, 2004, 139-179.

Eberhard, Philippe Lars: "Mise en musique du texte et musicalité de la parole dans la tragédie en musique ». In : Ibidem 55 (novembre 2016), 4-7.

Gier, Albert : Das Libretto. Theorie und Geschichte einer musikoliterarischen Gattung. Darmstadt : Wissenschaftliche Buchgesellschaft, 1998.

Girdlestone, Cuthbert : La tragédie en musique (1673-1750): considérée comme genre littéraire. Genève : Droz, 1972.

Gumbrecht, Hans Ulrich : "Musikpragmatik - Gestrichelte Linie zur Konstitution eines Objektbereichs ». In : Gier, Albert (éd.) : Oper als Text. Romanistische Beiträge zur Libretto-Forschung. Heidelberg : Carl Winter, 1986, 15-23.

Kintzler, Catherine : "La tragédie lyrique et le double défi d'un théâtre classique ». In : Christout, Marie-Françoise et al. (éds) : La tragédie lyrique (Les carnets du théâtre des Champs-Elysées). Paris : Cicéro, 1991, 51-63.

Le Cerfde La Viéville, Jean-Laurent: Comparaison de la musique italienne et de la musique françoise. Vol. 2. Bruxelles : François Foppens, 1705.

Leopold, Silke : Die Oper im 17. Jahrhundert. Laaber : Laaber, 2004.

Louvat-Molozay, Bénédicte : "Le théâtre français et le < partage du chant > ». In : Duron, Jean /

Doînel, Christophe (éds) : Grandes journées Lully. Naissance d'un roi. Versailles : Centre de Musique Baroque de Versailles, 2008, 69-88.

Lully, Jean-Baptiste : Atys. Tragédie mise en musique. Paris : Ballard, 1679.

Lully, Jean-Baptiste : "Armide. Tragédie en musique ». Éd. Lois Rosow. In : Lully, Jean-Baptiste : Euvres complètes. Éd. Jérôme de La Gorce et Herbert Schneider. Série III : Opéras, vol. 14. Hildesheim/Zürich/New York : Olms/Weidmann, 2003.

Lully, Jean-Baptiste : "Thésée. Tragédie en musique ». Éd. Pascal Denécheau. In : Lully, Jean-Baptiste : Euvres complètes. Éd. Jérôme de La Gorce et Herbert Schneider. Série III : Opéras, vol. 4. Hildesheim/Zürich/New York : Olms/Weidmann, 2010.

Lully, Jean-Baptiste : Thésée. Paris : Christophe Ballard, 1688. Mise en ligne le 4 novembre 2016, https://imslp.org/wiki/Th\%C3\%A9s\%C3\%A9e\%2C_LWV_51_(Lully\%2C_Jean-Baptiste) (consultation 10.01.2020).

Lully, Jean-Baptiste / Quinault, Philippe : Armide. Éd. N. Sceaux à partir de l'édition Ballard de 1686. Dernière révision le 11 mai 2014, http://nicolas.sceaux.free.fr/lully/LWV71Armide.pdf (consultation 10.01.2020).

Norman, Buford : Quinault, librettiste de Lully. Le poète des Grâces. Wavre : Mardaga, 2009.

Recueil général des opéras, représentés par l'Académie Royale de Musique, depuis son établissement. Paris : Ballard, 1703.

Sadie, Stanley (éd.) : The New Grove Dictionary of Opera. Vol. 3. London : Macmillan, 1992. 
Schneider, Herbert : «Lully, Jean-Baptiste ». In : MGG Online. Éd. Laurenz Lütteken. Kassel/ Stuttgart/New York 2016ff. Publié en novembre 2016, https:/www.mgg-online.com/mgg/ stable/13056 (consultation 18.06.2019). [Schneider 2016a]

Schneider, Herbert : "Tragédie lyrique - Tragédie en musique, Definition und Entstehung ". In : MGG Online. Éd. Laurenz Lütteken. Kassel/Stuttgart/New York 2016ff. Publié en novembre 2016, https://www.mgg-online.com/mgg/stable/13391 (consultation 18.06.2019). [Schneider 2016b] 